\title{
LA LITTÉRATURE MUSICALE CONGOLAISE: LA FÊTE DES MOTS
}

\author{
Lye M. Yoka
}

Institut National des Arts (INA), Kinshasa, République Démoncratique du Congo

\begin{abstract}
Résumé Il existe bon nombre d'écrits sur la musique congolaise moderne, notamment sur le plan de l'histoire et de la sociologie. Mais pas d'écrits spécialisés sur la litteralité des textes des chansons en termes stylistique, parémiologique et thématique. II faudrait en plus prendre en compte les survivances tenaces des traditions orales avec cette culture épicée de l' "éristique" qui est l'art de la dispute et de la palabre, assorti des artifices de la satire et de circonlocutions plus ou moins subversives. Finalement ce que les critiques "puristes" considèrent comme "paralittérature" (terme passablement dépréciatif!) c'est de la littérature en bonne et due forme.
\end{abstract}

MOTS CLÉS: LITTÉRATURE MUSICALE, ODYSSÉE ETÉPOPÉE DE LA RUMBA CONGOLAISE, CARACTĖRES TRANSPHRASTIQUE ET SYNTAGMATIQUE, TRAVESTISSEMENT DES PROFÉRATIONS THÉMATIQUES, RÉINVENTIONS PARÉMIOLOGIQUES

\begin{abstract}
Much has been written on modern Congolese music, particularly in terms of its history and sociology. However, there are no studies dedicated to the literary qualities of the song texts in stylistic, paremiological and thematic terms. In addition, when considering this body of music, the tenacious survival of oral traditions should be taken into account. Such traditions take in the vivid culture of the "eristic", the art of dispute and energetic discussion, accompanied by satirical turns and more or less subversive circumlocutions.

Finally, assert that what "purist" critics consider as "para-literature" (a rather deprecating term) is literature both in terms of its thematic and formal concerns.

KEYWORDS: MUSICAL LITERATURE, ODYSSEY AND EPIC OF THE CONGOLESE RUMBA, TRANSPHRASTIC AND SYNTAGMATIC CHARACTERS, DISGUISE OF THEMATIC PROFFERED, PAREMIOLOGICAL REINVENTIONS
\end{abstract}

\section{Mise au point}

C'est un évènement exceptionnel que le Prix Nobel de Littérature accordé le I3 octobre 2016 à l'artiste musicien américain Bob Dylan, 75 ans, "pour avoir créé, selon Sara Danius, Secrétaire Générale de l'Académie suédoise, dans le cadre de la grande tradition de la musique américaine, de nouveaux modes d'expression poétique». Bien entendu, comme il se doit à chaque prix Nobel, et plus spécialement à ce dernier, la controverse a été vive, les plus hostiles reprochant fondamentalement à l'Académie une sorte de confusion des genres... ${ }^{\mathrm{T}}$

Elie ARIE, "Pourquoi le prix Nobel à Bob Dylan est une grossière erreur", dans www.marianne.net/agora_blog, I6 novembre 20I6: "... Il ne s'agit pas de nier que certaines chansons, certains films sont des œuvres artistiques: il s'agit de nier que ce sont des œuvres littéraires. Bien sûr, il y a évidemment des relations entre la chanson, la poésie, la littérature, la musique et la danse, mais des rapports qui ne signifient pas identité: s'il y avait, 
Qu'à cela ne tienne : ce prix Nobel est pour nos littératures d'Afrique influencées de près ou de loin par les traditions orales, une aubaine, autrement dit la reconnaissance de la concrescence intime entre la parole, en tant que signe verbal diversifié, et la musique, en tant que poésie vivante.

N'est-il pas vrai que notre musique, la musique congolaise moderne, s'impose de plus en plus, et pas toujours là où on l'attend naturellement, c'est-à-dire dans les "ambiances " des discothèques, des bars, des concerts ou des shows. Cette musique $a$, depuis une cinquantaine d'années, pris d'assaut les cénacles des savants et les amphithéâtres des universitaires. Et quand nous écrivons «littérature congolaise», nous pensons bien évidemment aux deux Congo. S'il existe en effet des liens particulièrement forts, s'il existe de vrai "pont» (naturel et culturel) entre les deux pays, ce sont le fleuve et la musique. Au-dessus, au-delà des conjonctures et des conjectures souvent compliquées, toxiques et politiques des gouvernants. ${ }^{2}$

Habituellement, les critiques d'art, dans leur majorité, considèrent les prestations et les pratiques littéraires de la musique congolaise moderne, notamment les textes de la rumba, comme faisant partie de la "para-littérature», réservant le terme de «littérature " aux œuvres sanctuarisées des classiques français ou francophones. Par ailleurs, cette "paralittérature " a généralement été rangée dans le lot exotique de la "tradition orale».3

Autant d'écarts, voire d'excès de langage ! Les textes qui rythment la musique congolaise moderne, lorsqu'ils sont vraiment inspirés et travaillés, sont de l'ordre de la littérature à part entière, composante et préfiguration d'un art potentiellement "en totalité». Est-ce pour autant de la "littérature orale»? Non, cette littérature à part entière, prélude à la totalité de l'œuvre, est un va-et-vient dialectique et harmonique entre «littérature orale», «littérature manuscrite» (objet de l'analyse génétique et résultat de palimpsestes porteurs d'indices), littérature écrite, et chantée (par exemple sur des supports audiovisuels qui sont une nouvelle forme et force d'oralité mais électronique, ou dans des concerts qui sont des spectacles complets).

\section{Rumba congolaise : langages en folie}

On ne peut donc comprendre l'énergie et le génie de la rumba congolaise qu'à travers les mille facettes de cette épopée qui rythme l' après Deuxième Guerre Mondiale. 'Epopée' au sens premier d'une saga, d'une tradition orale vivante, effervescente, subliminale.

Les thèmes de la littérature musicale, empruntés souvent aux airs glamours de l'après-

en plus du prix Nobel de Littérature, un prix Nobel de Musique, lequel des deux aurait eu droit à Bob Dylan?" Plus loin l'auteur reconnait néanmoins avoir été "impressionné par le fait que trois grands romanciers que sont Alain Mabanckou, Salman Rushdie et Joyce Carol Oates (tous trois nobélisables !) soient d'un avis diamétralement opposé» au sien... Lire aussi : Antoine JACOB, «Les fissures du prix Nobel », dans L'Express, Paris, Nos 34I4 du 7 au I3 décembre 20I6, pp. 76-79.

2 Lye M. YOKA, «Pont sur le Congo: littérature et musique congolaises, même combat?», dans Combats pour la culture, Brazzaville, Les Editions Hemar, 20I2, pp. II8-I2I. 
guerre en Europe, notamment en France (Tino Rossi, Patrice et Mario, Line Renaud, Trenet, etc.) sont empreints de romantisme pétillant et du culte de la femme. Jamais le lingala, langue des deux capitales congolaises, Brazzaville et Kinshasa, n'a été autant la manifestation et l'expression populaires et sulfureuses faites de séduction et de fantasia ( Mino na yo pawuni penza», chante Kallé Jeef) ("Tes dents ont l'éclat d'une pierre précieuse») ; "Majos moke ya motema/ Yo fololo ya mabanzo/ Butu na moyi nakotuna/ Epayi okeyi nayebi te», chante Franco) («Petite Majos de mon cœur/fleur de mes pensées/ Nuit et jour je cherche / où tu as disparu»).

D'un autre côté cependant, le lingala a évolué sensiblement en un discours d'anomie, à travers sa parémiologie et ses «mbwakela», sorte de circonvolutions et de périphrases satiriques, comme une dynamique souterraine de résilience, de résistance populaire face aux rapports sociaux et sociétaux de plus en plus éprouvants.

Peut-être faudrait-il noter ici, depuis les années I950 jusqu'à aujourd'hui, le thème récurrent du «voyage» comme idéal d'évasion, mais par ailleurs comme description réaliste, initiatique des exodes compliqués vers ce que les habitants de 'l'arrière-pays' considèrent comme l'eldorado du Pool Malebo, Brazzaville et Kinshasa. Exemple, Antoine Moundanda: "Poto-poto mboka monene, solo Kinshasa, mpoto moindo" ("Poto-poto est certes une grande agglomération mais Kinshasa est une 'Europe noire'»); autre exemple, Antoine Wendo Kolosoy: "Wendo akomi Kinshasa», ballade sentimentale où le poète, ex-batelier, passe en revue et en souvenirs fredonnés, tous les confluents du fleuve Congo. Cette thématique du «voyage» inspiré principalement par une sorte de mystique du fleuve Congo a également connu ses jours de gloire avec des "tubes" à succès comme «Masuwa» (de Pamelo Mounk'a) ou «Ebale ya Zaïre» de Lutumba Simaro, autant de poèmes empreints de nostalgie des « départs " ainsi que des «adieux» mélancoliques des bateaux, avec toujours en toile de fond les amours disloqués...

Enfin, cette épopée prend de temps en temps des allures philosophiques radicales sur les fondamentaux mythologiques et historiques négro-africains, avec des accents profonds de doute métaphysique ou de militantisme mobilisateur. Exemples : "Nakomitunaka» de Kiamwangana Mateta Verckys, série d'interrogations sur les malentendus des enseignements bibliques inadaptés par rapport aux réalités bantoues. "Pouvoir noir de Franco» est une ode à l'énergie et au génie négro-africains. "Mi Angola» de Sam Mangwana vante le combat héroïque pour la libération de l'Angola ; de même que "Congo monene" de Franklin Boukaka, hommage au 'grand Congo de Lumumba' (c'est-à-dire les deux Congo ensemble), etc.

\section{Une littérature de la totalité}

Cette littérature qui s'énonce totale, "joue» la musique, au sens plein de "mise en scène", avec ses personnages, ses rituels, ses célébrants, ses intrigues et ses mythes, ses gesticulations... Il s'agit d'une littérature musicale contextualisée, chorégraphiée, scénographiée, scénarisée, théâtralisée même : à force de signes verbaux et non-verbaux, à force de travestissement et de jeux de rôles, à force de signes kinésiques et proxémiques, à force des va-et-vient de la fiction à la réalité et vice versa ; bref, avec des figures de styles 
à la fois classiques et spécifiques, conventionnels et recréés; et donc avec ses oxymorons propres. On pourrait décrire ces figures de style de la façon détaillée suivante:

Travestissement diégétique : il s'agit, au cours de l'énonciation, de l'ambivalence du «JE»: le travestissement des rôles prête habituellement la parole et la voix aux hommes afin de dénoncer des leçons morales, en lieu et place des femmes elles-mêmes. Exemple: la chanson «Bina na ngai na respect» ('Danse avec moi avec respect') composée et chantée par Ntesa Dalienst, au cours de laquelle une femme seule sur la piste de danse d'un concert, repousse les avances empressées et maladroites d'un courtisan.

«Liboso ya kosala likambo bangaka makambo/ Naye kobina naye kosepelisa nzoto/ Nazali mwasi ya bato (...)/Opesi ngai mbote ngai nandimi/ Ekomi lisusu tobina (...) ngai naboya te/ Oluki ngai makango wana nde 'jamais'»

(«Avant d'agir, réfléchis et prends garde/ Je suis là pour m’éclater et faire plaisir à mon corps/ Mais je suis l'épouse d'un époux/ Tu me salues, je te salue/ Tu m'invites à danser, pourquoi pas? I Tu dragues et harcèles, je dis 'jamais !'»)

Substitution paradigmatique: c'est le remplacement des signes verbaux par des signes non-verbaux; la palabre est remplacée par la pantomime. Exemple: "Catherine ndoki» ("Catherine sorcière») de Franco Luambo. Une rivale dénonce les manœuvres dilatoires d'une concurrente qui s'en est allée consulter en cachette un féticheur pour que le concubin commun l'abandonne au profit de l'intrigante. Après un couplet narratif sur ces funestes manœuvres, la voix de la rivale lésée, par le biais du chanteur-narrateur, se transforme en imitation du féticheur avec des formules sous forme de borborygmes énigmatiques et hiératiques.

En fait, en observant de près toutes ces pratiques théâtralisées, on se rend compte combien elles nous rapprochent des techniques du conte africain avec un même rôle, celui généralement du narrateur-actant, à la fois protéiforme et multiforme sur scène.

Ici, le cas le plus connu est celui de la chanson «Course au pouvoir» de Franco Luambo; c'est une sévère diatribe de Franco, à la première personne du pronom et du sujet, contre l'un de ses collaborateurs qui l'avait traité d'escroc et de "faux millionnaire». Si la première partie de la chanson est un propos incendiaire, à la limite de la trivialité («Opolaki olumbi solo/ Obimaki soyi ya mobesu») ("Toi tout vivant, tu étais déjà en putréfaction nauséabonde/ tu déversais même des saletés de crachats "), la seconde partie met en scène l'affrontement direct, à travers les sons et les tons martiaux des instruments de musique comme autant d'armes brandis : la guitare-solo entre les mains de Franco d'une part, et le saxophone de Kiamwangana d'autre part. Franco et Verckys "jouent à se faire peur", au figuré. Guitare et saxophone «croisent le fer» dans une comédie musicale burlesque...

Apposition transphrastique et juxtaposition syntagmatique: il s'agit d'une expérience de la troupe théâtrale de l'Institut National des Arts en 2000.4 Dans un processus 
inédit de réécriture scénique, le metteur en scène a collé bout à bout, sur base des différents répertoires de l'artiste Koffi Olomide: des textes, des airs, des aphorismes, des figures chorégraphiques, des saynètes reliées, tout cela extrait des compositions musicales originales, pour une intrigue refaite sur le thème de l'amour impossible d'un couple de jeunes kinois. Titre du spectacle : «Koffi-Papa-Tout».

"Parodie » et "jeu de masques ". Il faudrait prendre "parodie» au sens premier et au sens dérivé: celui d'une part d'un "texte composé pour être chanté sur une musique connue ", 5 et celui d'autre part d'une imitation satirique, d'un discours parallèle, indirect, rusé et oblique. La procédure, dans certaines versions musicales congolaises, consiste à louer les services d'un "commissionnaire-mercenaire» parmi les artistes compositeurs les plus doués, et lui commander une chanson suffisamment «létale» en termes de mbwakela empoisonnés. Et donc, à défaut d'une confrontation directe entre rivales, chacune de son côté se camoufle derrière ce "commissionnaire-mercenaire», cet épouvantail, et fait lancer des piques assassines. Exemples : la première rivale ayant décoché, via l'artiste compositeur Lutumba Simaro, une salve particulièrement empoisonnée dans la chanson «Bisalela»

("Gesticulations »), l'adversaire répliqua aussitôt avec la même vigueur et la même verdeur, par le même "porte-parole» et à travers des répliques venimeuses, par exemple «Ngai namoni kaka», "Bodoutaka» (de la racine «douter». Traduction: «avez-vous besoin de douter encore?»). Par exemple encore: «Faute y a commerçant» («C'est la faute du commerçant». ${ }^{6}$ )

Recours à la parémiologie éristique : la parémiologie, dans ce contexte-ci, est l'usage des proverbes, souvent inventés ou actualisés pour les besoins de la cause, comme techniques de provocation, comme leçons morales ou comme choc des mots («l'éristique» étant, dans la tradition rhétorique grecque, " l'art de la dispute»). Exemples :

— «ve dire toza na système ya lifelo ; kasi motu akozika te" (A vrai dire, nous sommes au cœur du système de l'enfer; mais il n'y a, pour personne, le risque d'être brûlé) : Koffi Olomide;

— «Mvula epanzaka matanga kasi zando te» (La pluie disperse une veillée de deuil mais pas un marché) : Papa Wemba;

— «Bikela-kela ezali lokola mapapu ya kipungu-pungu; soki oluki okanga ye na mapapu, okotikala kaka na putulu ya langi na maboko» ( L'œuvre d'art ressemble aux ailes de la libellule: c'est vain que vous cherchez à attraper l'insecte par ses ailes ; il ne vous restera sur les mains que de la poussière colorée» : Lutumba Simaro.

5 Cf. Wiktionnaire, http://wiktionary.org

6 "Faute ya commerçant» : le commerçant est la métaphore du concubin commun à deux rivales : il partage et il est partagé à égalité entre les deux. Le commerçant vend, sans état d'âme, les mêmes pagnes des mêmes motifs et des mêmes tarifs aux mêmes clientes. D'après le compositeur Lutumba, le concubin, l' "ambianceur» 'vend' et ' partage', sans état d'âme, ses sentiments à prix égal... 


\section{"Lire la musique " $^{7}$ : l'état actuel des approches descriptives et critiques}

n peut se féliciter aujourd'hui de l'émergence de nombreuses recherches scientifiques sur la musique congolaise moderne. Or les tendances habituelles de ces analystes et critiques ont été ou sont consignées encore soit sur la synchro-diachronie (Michel Lonoh, Sylvain Bemba, Manda Tchebwa, $\mathrm{Mfumu}^{8}$ ), soit sur des monographies biographiques (Mfumu, Mayengo, Nimy, Tshonga Onyiumbe ${ }^{9}$ ), soit sur les interférences ethno-musicologiques (Philippe Kanza ${ }^{10}$ ), soit encore sur la sociologie (Tshonga Onyiumbe, Léon Tsambu ${ }^{\mathrm{II}}$ ), soit enfin sur les théories et les enquêtes concernant l'écoute et la réception (Bob White et Lye Yoka, Ribio Nzeza $\left.{ }^{12}\right)$. Peu de chercheurs se livrent à une analyse littéraire proprement dite de cette musique dans ses formes et styles, dans ses marqueurs locutoires, illocutoires, et perlocutoires ; mais aussi dans ses prolongements sémiotiques et spectaculaires.

La tâche du sémiologue devant un spectacle justement, n'est-elle pas, ainsi que le décrit Anne Ubersfeld à propos de la représentation dramaturgique, «de faire éclater par des pratiques sémiotiques et textuelles le discours dominant, le discours appris, celui qui interpose entre le théâtre et la représentation tout un écran invisible de préjugés, de personnages, et de «passions", le code même de l'idéologie dominante ${ }^{\mathrm{I}}{ }^{3}$

Y a-t-il meilleure façon de décrire justement l' "ambiance» festive, insolite, pétillante, entre l'instant de composition du texte et son éclosion, son explosion en concert public? Y a-t-il meilleure approche de cerner le concept de « discours dominant» ou d' "idéologie

Toutes proportions gardées, "Lire la musique» pourrait être mis en comparaison par rapport à l'essai d'Anne UBERSFELD, Lire le théâtre, Paris, Editions sociales, I977 . L'auteur écrit, à propos de théâtre : «...C'est la spécificité du texte de théâtre qui est la première question posée, la question essentielle ; trouver les éléments de réponse, c'est peut-être échapper à la fois au terrorisme textuel et au terrorisme scénique, à ce conflit entre celui qui privilégie le texte littéraire et celui qui, aux prises avec sa seule pratique dramaturgique, fait fi de l'instance scripturale».

8 Lire entre autres publications :

- Sylvain BEMBA, Cinquante ans de musique du Congo-Zaïre (1920-1970). De Paul Kamba à Tabu Ley, Paris, Présence Africaine, 1984 .

- MANDA TCHEBWA, Terre de la chanson, la musique zaïroise: hier et aujourd'hui, Louvain-La-Neuve, DUCULOTAfrique Editions, I996.

- MFUMU, La musique congolaise du XXe siècle, Brazzaville, Beau D Pro, 2006.

9 Lire «Musique rétro", dans la revue Rétro, Nos I et 2, Brazzaville, Atelier Beaudley, 2013.

Io KANZA MATONDO, Musique zaïroise moderne. Situation actuelle et perspectives, Kinshasa, Publications du Conservatoire National de Musique et d'Art Dramatique (CNMA), I972.

II Lire par exemple:

- Yvon Laurier NGOMBE, Rumbissimo. Rumba, droit et business, Paris, BOD/ Books-On-Demand, 2013.

- Léon TSAMBU, Luttes symboliques et enjeux de domination sur l'espace de la musique populaire à Kinshasa. Critique praxéologique des sociabilités de la scène musicale kinoise. Thèse de doctorat, Faculté de Sciences sociales. Université de Kinshasa, 2012.

I2 - Bob WHITE et Lye M. YOKA, Musique populaire et société. Une ethnographie de l'écoute, Paris, L'Harmattan, 2010. - Ribio NZEZA, Réception des chroniques musicales télévisées à Kinshasa (RD.Congo). Du divertissement à la construction des perspectives d'identités socioprofessionnelles chez les jeunes. Thèse de doctorat, Faculté des Communications sociales. Université Catholique du Congo, 2016. 
dominante", autrement dit de "soft power " ${ }^{\mathrm{I} 4}$ à travers le pouvoir politique de la musique populaire, au service ou à contre-courant des gouvernants ou des régimes en place.

\section{La tâche de l'Institut National des Arts-Kinshasa}

C'est d'ailleurs pourquoi, depuis près de cinq ans, autour du Festival Rumba Parade (en partenariat avec l'Agence EALE CMCT, et la Délégation Wallonie-Bruxelles à Kinshasa), les chercheurs et les praticiens de l'Institut National des Arts (INA) procèdent à la promotion de la musique congolaise moderne comme patrimoine culturel immatériel, par des opérations suivantes :

I. identification des chansons emblématiques du répertoire congolais ;

2. identification des paramètres originaux d'analyse et de sélection ;

3. traduction en langue française des textes littéraires de ces chansons en langues nationales ;

4. transcription en partitions musicologiques des textes originaux;

5. publication des anthologies sur des morceaux ainsi choisis ;

6. réorchestration et exécution publique, par l'orchestre de l'INA, des morceaux choisis, avec des instruments à la fois classiques (occidentaux) et traditionnels; mais aussi, dans la mesure du possible, avec des danses et des «ambiances» reconstituées.

Que vise l'INA par cette démarche mémorielle et re-créative? D'abord, à court et à moyen terme, conserver et revaloriser ce patrimoine culturel immatériel et essentiel; ensuite, à moyen et à long terme, arriver, pourquoi pas, à constituer des anthologies de haute référence, du point de vue à la fois littéraire et musical. Mais arriver en même temps à inscrire la rumba congolaise sur les listes respectives du patrimoine culturel national et mondial. ${ }^{15}$

En fin de compte, le rêve des chercheurs de l'INA est de capitaliser toute cette créativité artistique totale et intégrée, en termes de valeur ajoutée: valeur du devoir de mémoire, valeur de l'éducation nationale (au sens plein du terme, et pas seulement celle de 'l'instruction publique'), ainsi que valeur du dialogue interculturel et de la cohésion nationale.

I4 Lire à ce propos:

- Frédéric MARTEL, Mainstream. Enquête sur la guerre globale de la culture et des médias, Paris, Flammarion, 2010.

- Lye M. YOKA, «Musique congolaise et pouvoir», dans Combats pour la culture, op.cit., pp. I49-I62.

I5 En date du 3 août 2or6, le Ministre de la Culture et des Arts de la RD.Congo a signé deux Arrêtés, l'un No 032/ CAB/MIN/CA/DIRA/SA/20I6, et l'autre No o36/CAB/MIN/CA/DIRA/YMM/20I6, portant respectivement:

- d'une part, création de la «Commission Nationale en charge de la Promotion pour l'Inscription de la Rumba sur la Liste du Patrimoine Culturel Immatériel de la RD.Congo, et sur la Liste du Patrimoine Culturel Immatériel de l'Humanité (CNIPR); et

- d'autre part, nomination des membres de cette Commission, avec comme Président Pr Lye M. YOKA. 